\title{
Nutritional intervention in patients with periodontal disease: clinical, immunological and microbiological variables during 12 months
}

\author{
Axel Jenzsch ${ }^{1}$, Sigrun Eick ${ }^{2}$, Fausi Rassoul ${ }^{3}$, Regina Purschwitz ${ }^{1}$ and Holger Jentsch ${ }^{1}$ \\ ${ }^{1}$ Department of Conservative Dentistry and Periodontology, University of Leipzig, Liebigstraße 57, D-04103 Leipzig, Germany \\ ${ }^{2}$ Department of Oral Microbiology, Institute of Medical Microbiology, University of Jena, Jena, Germany \\ ${ }^{3}$ Institute of Laboratory Medicine, Clinical Chemistry and Molecular Diagnostics, University of Leipzig, Leipzig, Germany
}

(Received 5 September 2007 - Revised 25 June 2008 - Accepted 3 July 2008 - First published online 20 August 2008)

The role of nutrition in onset, progression and treatment of periodontitis has not been thoroughly evaluated. In the present prospective clinical study, we investigated the influence of a nutritional intervention on changes in clinical, microbiological and immunological periodontal variables during a period of 12 months in patients with the metabolic syndrome and chronic periodontitis. Twenty female subjects with the metabolic syndrome and mild to moderate chronic periodontitis participated in a guided nutritional intervention programme. Examinations were assessed before, and at 2 weeks, 3, 6 and 12 months after intervention. Clinical measurements included probing depth, Löe and Silness gingival index and QuigleyHein plaque index. In gingival crevicular fluid, periodontopathogens, levels of IL-1 $\beta$ and IL- 6 as well as the activity of granulocyte elastase were determined. In stimulated saliva, antioxidative and oxidative variables were measured. After 12 months the following significant changes could be observed: reduction of clinical probing depth $(2.40$ v. $2.20 \mathrm{~mm} ; P<0.001)$, reduction of gingival inflammation (gingival index 1.13 v. 0.9 ; $P<0.001)$, reduced concentrations of IL-1 $\beta(4.63 v .1 .10 \mathrm{pg} / \mathrm{ml}$ per site; $P<0.001)$ as well as IL-6 $(1.85 v .0 .34 \mathrm{pg} / \mathrm{ml} \mathrm{per}$ site; $P=0.022)$ in gingival crevicular fluid. Bacterial counts in gingival crevicular fluid as well as oxidative and antioxidative variables in saliva showed no significant changes. Only salivary catalase showed a tendency to lower values. These findings indicate that in patients with the metabolic syndrome wholesome nutrition might reduce inflammatory variables of periodontal disease and promote periodontal health.

Periodontitis: Diet: Metabolic syndrome

Periodontitis is the most common chronic inflammatory disease caused by an interaction of a specific bacterial flora with the host. Many modifiable and non-modifiable risk factors, among them obesity, can modify the individual predisposition to periodontal disease ${ }^{(1)}$. Obesity has significant metabolic, systemic immune and inflammatory effects and may increase the host's susceptibility to periodontal disease through its impact on metabolic and immune parameters ${ }^{(2)}$. It is known that obesity is strongly linked to a raised subclinical inflammatory status and type 2 diabetes $^{(3)}$.

The role of nutrition as a possible modifiable systemic factor in periodontal disease onset, progression and treatment has not been thoroughly examined. Only a few studies have examined relationships between diet and periodontitis and suggest that a high intake of fruits and whole-grain products is associated with a lower prevalence of periodontitis ${ }^{(4,5)}$. Individuals with poor dietary habits were found to be three times more likely to have periodontitis than individuals with good dietary habits, independent of other major risk factors for periodontitis ${ }^{(6)}$. An analysis of the third National Health and Nutrition Examination Survey data suggest a protective association between a good overall quality of diet and the prevalence of periodontitis ${ }^{(7-9)}$.

Nutrition might influence the development of oral biofilms, inflammation and immune response, tissue metabolism, wound healing and quality and quantity of saliva ${ }^{(10,11)}$. For example, the release of cytokines, such as IL-1 $\beta$ and IL-6, which are implicated in periodontal tissue destruction, can be modulated by nutrients ${ }^{(12)}$.

Furthermore, changes in granulocyte functions occur in obesity $^{(13)}$. Polymorphonuclear granulocytes have an important role in the maintenance of gingival and periodontal health. During their interactions with micro-organisms, polymorphonuclear granulocytes develop a potential destructive role ${ }^{(14)}$. The result of their activation as a reaction on the microbial attack is, among others, an increased release of elastase $^{(15)}$. Neutrophilic elastase has been suggested to be a potential indicator of the degree of periodontal disease and disease progression ${ }^{(16)}$.

Nutrients, such as vitamin E, vitamin C, carotenoids, polyphenols, glutathione and trace elements, can contribute directly and indirectly to the robustness of antioxidant defences of the host ${ }^{(17)}$. Oxidative stress is involved in the pathogenesis of a number of diseases, including periodontitis $^{(18)}$. An adequate intake of antioxidants and PUFA may be important for preventing oxidative stress ${ }^{(19)}$. However, the extent of dietary influence upon salivary antioxidant status is unclear $^{(20)}$.

The purpose of the present study was to assess the effects of a guided nutritional intervention over 1 year on clinical, 
immunological and microbiological variables in patients with mild to moderate chronic periodontitis and the metabolic syndrome.

\section{Experimental methods}

\section{Patients and nutrition intervention}

Twenty women (aged 55.0 (SD 10.9) years) participated in the present prospective clinical study. After being informed on the purpose of the study, written informed consent was obtained from all patients. The study has been independently reviewed and approved by the local ethical committee of the University of Leipzig.

The inclusion criteria were as follows: at least fourteen teeth in occlusion; untreated mild or moderate chronic periodontitis; unmodified conventional oral hygiene and no current periodontal therapy, non-smoker; diagnosed metabolic syndrome; BMI $>25 \mathrm{~kg} / \mathrm{m}^{2}$.

Exclusion criteria were: severe adiposity with a BMI $>40 \mathrm{~kg} / \mathrm{m}^{2}$; intake of lipid metabolism-regulating drugs; diabetes mellitus with administration of insulin and/or antidiabetic therapy; intake of antibiotics during the last 3 months; special dietary patterns, such as vegetarian diet; systemic diseases other than the metabolic syndrome.

All participants were integrated in a nutritional intervention programme assisted by nutritionists. The consultations with the nutritionists included the evaluation of individual dietary habits, lectures about diet and health, dietary habits, specificity of single groups of food, instructions for wholesome nutrition, the preparation of food in groups as well as continuous individual sessions. The consultations started directly after the baseline examination and were perpetuated the first 2 months every 2 weeks and then every 2 months. Participants were deemed to be highly compliant if they regularly attended and cooperated with the consultations. During an intervention period of 1 year, the participants changed their dietary habits from an average German mixed diet towards wholesome nutrition. Wholesome nutrition has the following general features: (1) preference of food of plant origin; (2) preference of food processed as little as possible; (3) plentiful consumption of unheated fresh food; (4) careful preparation of meals from fresh foods; (5) the sparse use of fat ${ }^{(21)}$. This diet is mainly composed of vegetables, fruits, whole-grain products, potatoes, legumes and dairy products. The consumption of meat, fish and eggs is limited to one or two portions per week. The recommendations of wholesome nutrition are given in Table 1.

\section{Clinical periodontal measurements}

The clinical data and samplings were obtained before nutritional intervention and 2 weeks, 3, 6 and 12 months later. At all visits, gingival crevicular fluid and stimulated whole saliva were collected first and then clinical variables were recorded. Clinical probing depth was determined at four sites per tooth. The extent of gingival inflammation was determined by the criteria of the gingival index according to Löe \& Silness ${ }^{(22)}$. The gingival index differentiates the gingival inflammation into the score 0 (normal), 1 (slight inflammation), 2 (moderate inflammation) and 3 (severe inflammation). Oral hygiene status was assessed using the modified Quigley-Hein plaque index ${ }^{(23)}$. This index assessed the plaque formation on the teeth into the score 0 (no plaque), 1 (separate spots of plaque at the cervical margin of the tooth), 2 (thin continuous band of plaque up to $1 \mathrm{~mm}$ at the cervical margin of the tooth), 3 (band of plaque wider than $1 \mathrm{~mm}$ but covering less than one-third of the tooth), 4 (plaque covering at least one-third but less than two-thirds of the crown) and 5 (plaque covering two-thirds or more of the crown). The clinical assessments were carried out with a manual periodontal probe with $1 \mathrm{~mm}$ graduations (UNC 15; Hu-Friedy, Chicago, IL, USA). All measurements and sample collections were made by a calibrated examiner.

\section{Determination of microflora and immunological variables in gingival crevicular fluid}

Before each clinical examination, gingival crevicular fluids were obtained from two selected sites with a probing depth $\geq 3 \mathrm{~mm}$ for each subject. For the following examinations the same sites were chosen for sampling. Supragingival plaque was carefully removed with a curette and the site was gently dried with an air stream. Periopaper (Pro flow ${ }^{\mathrm{TM}}$, Amityville, NY, USA) was inserted into the gingival crevice and kept there for $2 \mathrm{~min}$. Contamination with blood or saliva was avoided. Thereafter, strips were stored in $100 \mu \mathrm{l}$ of $0.5 \mathrm{M}-\mathrm{NaCl}$ at $-18^{\circ} \mathrm{C}$ until analysis. Immediately before the analysis, all samples were eluted for $2 \mathrm{~h}$ at room temperature in $500 \mu \mathrm{l}$ of $0.5 \mathrm{M}-\mathrm{NaCl}$.

Elastase activity was determined by means of a chromogenic substrate specific for granulocyte elastase (1 mM- $N$-methoxysuccinyl-Ala-Ala-Pro-Val-pNa). In brief, to $90 \mu$ l eluate of gingival crevicular fluid sample, $10 \mu$ l substrate was added. The absorbance at $405 \mathrm{~nm}$ was measured immediately in a microplate reader. After incubation for $30 \mathrm{~min}$ at $37^{\circ} \mathrm{C}$ the measurement was repeated. The readings were

Table 1. Recommendations of wholesome nutrition for food consumption ${ }^{(21)}$

\begin{tabular}{llll}
\hline How much and how often to consume & & \\
\hline Generously & Moderately & \multicolumn{1}{c}{ Seldom } & Avoid \\
\hline Vegetables & Nuts & Canned food & Isolated sugars \\
Fruits & Oil seeds & White flour products & Sweets \\
Wholemeal products & Native oils & Refined fats & Isolated substances \\
Potatoes & Butter & Meat products & Ready-to-eat highly processed products \\
Legumes & Milk (products) & Alcohol & \\
Spices & Meat, fish, eggs & & \\
Water & Salt & & \\
\hline
\end{tabular}


subtracted from the $0 \mathrm{~h}$ value (difference of optical density at $405 \mathrm{~nm}$ ) and activity was expressed as arbitrary units per site.

IL-1 $\beta$ and IL-6 were analysed from $100 \mu$ l eluate by using a commercially available ELISA (Human IL-1beta CytoSets ${ }^{\mathrm{TM}}$, Human IL-6 CytoSets $^{\mathrm{TM}}$; BioSource International Inc., Camarillo, CA, USA). These assays were used according to the manufacturer's instructions using recombinant human standards. Through an antibody-antigen-antibody reaction with a direct coupled enzymic reaction, small amounts of cytokines were detectable. Results for IL-1 $\beta$ and IL-6 are reported as $\mathrm{pg} / \mathrm{ml}$ per site.

For the microbial analysis, $100 \mu$ l of the eluate from the paper strips were used for DNA extraction and purification with a commercial kit (Genomic Mini; A\&A Biotechnology, Gdynia, Poland). After isolation, DNA was stored at $-20^{\circ} \mathrm{C}$ until analysis by quantitative real-time PCR. The real-time PCR amplification was performed in a total reaction mixture volume of $20 \mu \mathrm{l}$. Each sample was quantitatively analysed for Tannerella forsythia, Treponema denticola, Porphyromonas gingivalis, Aggregatibacter actinomycetemcomitans, Prevotella intermedia and Fusobacterium nucleatum. The reaction mixture contained $7.9 \mu \mathrm{l}$ distilled water, $2 \mu \mathrm{l} \mathrm{PCR}$ puffer, $2 \mu \mathrm{l}$ dNTPs, $2 \cdot 1 \mu \mathrm{l} \mathrm{MgCl}_{2}, 1 \mu \mathrm{l}$ SYBR Green, $1 \mu \mathrm{l}$ Taq polymerase, $2 \mu \mathrm{l}$ of the pathogen-specific primer and $2 \mu l$ of purified DNA from gingival crevicular fluid samples. The primers used for Porphyromonas gingivalis, Tannerella forsythia, Prevotella intermedia and Treponema denticola have been described by Ashimoto et al. ${ }^{(24)}$, for A. actinomycetemcomitans by Rudney et al. ${ }^{(25)}$ and for $F$. nucleatum by Fouad et al. ${ }^{(26)}$. The amplification was performed in the Rotor-Gene $^{\mathrm{TM}} 2000$ real-time cycler (Corbett Research, Sydney, Australia) and the complete PCR sample data of each periodontopathogen were quantitatively analysed using the real-time PCR software of the Rotor-Gene ${ }^{\text {TM }} 2000$ system.

\section{Saliva collection and assays for oxidative and antioxidative variables}

The stimulated saliva samples were obtained at each date of examination under standardised conditions while chewing paraffin wax for $5 \mathrm{~min}$. The samples were centrifuged at $3000 \mathrm{~g}$ and $2-4^{\circ} \mathrm{C}$ for $15 \mathrm{~min}$; the supernatant fractions were stored at $-18^{\circ} \mathrm{C}$ until analysis.

The assay used for determining the activity of myeloperoxidase has been described by de Mendez et al. ${ }^{(27)}$. The substrate includes Triton-X-100, $o$-dianisidine and $\mathrm{H}_{2} \mathrm{O}_{2}$ in sodium citrate buffer. The absorbance at $450 \mathrm{~nm}$ was measured immediately in a microplate reader. After incubation for $30 \mathrm{~min}$ at $37^{\circ} \mathrm{C}$ the measurement was repeated. These measurements were also performed including sodium azide as an inhibitor of myeloperoxidase ${ }^{(28)}$. The readings of substrate and sample were subtracted from the values including additional inhibitor.

Lipid peroxidation products were assayed using TCA, thiobarbituric acid and deoxylsulfate as substrate ${ }^{(29)}$. A standard was also compounded. After incubation for $25 \mathrm{~min}$ at $95^{\circ} \mathrm{C}$ and cooling down in ice, the reaction was stopped with a mix of water and butanol. After shaking for $20 \mathrm{~min}$ and centrifugation at $1000 \mathrm{~g}$ for $7 \mathrm{~min}$ the absorbance was measured at a wavelength of $586 \mathrm{~nm}$.
Glutathione peroxidase activity was measured according to Lawrence \& Burk ${ }^{(30)}$. PBS solution including EDTA, glutathione, NADPH and glutathione reductase was used as substrate. The change in absorbance at $304 \mathrm{~nm}$ was monitored spectrophotometrically for $1 \mathrm{~min}$ and the mean substrate blank was subtracted from the sample readings.

The activity of catalase was assayed using the method of Beers \& Sizer ${ }^{(31)}$. The decomposition of $\mathrm{H}_{2} \mathrm{O}_{2}$ was monitored spectrophotometrically at $240 \mathrm{~nm}$ for $1 \mathrm{~min}$. The values of substrate without sample were subtracted from the sample readings. For all variables the rate of activity is expressed as arbitrary units per site.

\section{Statistical analysis}

Due to normal distribution tested by the KolmogorovSmirnov test, the values of the clinical variables are expressed as means and standard deviation. Overall differences were tested using general linear model variance analysis; differences between two appointments were tested using the $t$ test. The differences of the microbiological and immunological variables during the intervention period were tested using the non-parametric Friedman test, because no normal distribution was calculated. In the case of a significant Friedman test, differences between two appointments were assessed using the Wilcoxon test. Significance was set at $P \leq 0 \cdot 05$.

\section{Results}

Twenty female subjects (mean age: $55 \cdot 0$ (SD 10.9) years) completed all evaluations during the nutritional intervention period of 12 months. Only compliant subjects were included in the analysis. Non-compliant subjects were a priori excluded from the study. The baseline characteristics of the subjects are shown in Table 2. The clinical measurements are given in Table 3. Regarding the whole oral cavity, the mean probing depth was significantly reduced from $2.40 \mathrm{~mm}$ at baseline to $2.20 \mathrm{~mm}$ after 12 months $(P<0.001)$. The probing depth of the collected sites showed no significant changes during the

Table 2. Baseline characteristics of the study participants

(Mean values and standard deviations)

\begin{tabular}{lcc}
\hline & \multicolumn{2}{c}{ Baseline } \\
\cline { 2 - 3 } Variable & Mean & SD \\
\hline Age (years) & 55.0 & $10 \cdot 9$ \\
Body weight $(\mathrm{kg})$ & 77.6 & 12.7 \\
Height $(\mathrm{cm})$ & $164 \cdot 0$ & $7 \cdot 0$ \\
BMI $\left(\mathrm{kg} / \mathrm{m}^{2}\right)$ & 28.9 & 4.3 \\
Waist:hip ratio & $0 \cdot 88$ & 0.05 \\
Total body fat $(\%)$ & 35.7 & 4.15 \\
Systolic blood pressure $(\mathrm{mmHg})$ & 135.0 & 16.4 \\
Diastolic blood pressure $(\mathrm{mmHg})$ & 85.5 & 9.89 \\
Serum TAG (mmol/l) & 1.5 & 0.6 \\
Serum total cholesterol $(\mathrm{mmol} / \mathrm{l})$ & 6.0 & 1.3 \\
Serum LDL-cholesterol $(\mathrm{mmol} / \mathrm{l})$ & 4.0 & 1.2 \\
Serum HDL-cholesterol $(\mathrm{mmol} / \mathrm{l})$ & 1.5 & 0.3 \\
Serum homocysteine $(\mu \mathrm{mol} / \mathrm{l})$ & 9.8 & 2.9 \\
Serum fasting glucose $(\mathrm{mmol} / \mathrm{l})$ & 5.0 & 0.7 \\
Serum uric acid $(\mu \mathrm{mol} / \mathrm{l})$ & 414.0 & 60.0 \\
Plasma C-reactive protein $(\mathrm{mg} / \mathrm{l})$ & 3.6 & 3.2 \\
\hline
\end{tabular}


Table 3. Clinical variables at baseline and during the nutritional intervention period (Mean values and standard deviations)

\begin{tabular}{|c|c|c|c|c|c|c|c|c|c|c|c|}
\hline \multirow[b]{2}{*}{ Variable } & \multicolumn{2}{|c|}{ Baseline } & \multicolumn{2}{|c|}{2 weeks } & \multicolumn{2}{|c|}{3 months } & \multicolumn{2}{|c|}{6 months } & \multicolumn{2}{|c|}{12 months } & \multirow[b]{2}{*}{$P^{*}$} \\
\hline & Mean & SD & Mean & SD & Mean & SD & Mean & SD & Mean & SD & \\
\hline \multicolumn{12}{|c|}{ Whole oral cavity } \\
\hline $\mathrm{PD}(\mathrm{mm})$ & 2.40 & 0.40 & 2.50 & 0.40 & $2 \cdot 40$ & 0.40 & $2 \cdot 30$ & 0.40 & $2 \cdot 20 \dagger$ & 0.50 & $<0.001$ \\
\hline GI & $1 \cdot 13$ & 0.50 & $1 \cdot 19$ & 0.38 & 1.01 & 0.51 & 0.93 & 0.51 & $0.90 \ddagger$ & 0.53 & 0.002 \\
\hline QHI & $1 \cdot 14$ & 0.64 & 0.99 & 0.63 & 1.09 & 0.46 & 1.05 & 0.41 & 1.07 & 0.61 & 0.703 \\
\hline \multicolumn{12}{|c|}{ Sites of collection for gingival crevicular fluid } \\
\hline $\mathrm{PD}(\mathrm{mm})$ & 3.20 & 1.00 & 3.20 & 1.00 & 3.00 & 1.00 & 3.00 & $1 \cdot 11$ & 3.00 & $1 \cdot 11$ & 0.753 \\
\hline Gl & 1.13 & 0.64 & 0.99 & 0.63 & 1.09 & 0.46 & 1.05 & 0.41 & $1.07 \dagger$ & 0.61 & $<0.001$ \\
\hline
\end{tabular}

PD, probing depth; GI, gingival index; QHI, modified Quigley-Hein index for oral hygiene status.

* General linear model variance analysis.

Mean value was significantly different from that at baseline: $\dagger P<0.001, \ddagger P=0.029$ (paired $t$ test).

nutritional intervention period. Oral hygiene status, expressed by the Quigley-Hein index, showed no changes during the intervention period. We observed a significant reduction of the gingival index from 1.13 at baseline to 0.90 after 12 months, respectively $(P<0.001)$. In accordance with the gingival index the concentration of IL-1 $\beta$ in gingival crevicular fluid decreased continuously over the nutritional intervention period from $4.63 \mathrm{pgml}$ per site at baseline to $1.10 \mathrm{pgml}$ per site after 12 months $(P<0 \cdot 001)$ (Fig. 1). Furthermore, the concentration of IL-6 was also significantly reduced $(P=0 \cdot 022)$. A decrease from $1.85 \mathrm{pgml}$ per site at baseline to $0.34 \mathrm{pgml}$ per site after 12 months could be observed (Fig. 2). No significant changes could be found for the activity of granulocyte elastase in gingival crevicular fluid (data not shown).

In stimulated whole saliva the oxidative variables (activity of myeloperoxidase and level of lipid peroxidation products) were determined. The result for myeloperoxidase was $0 \cdot 118$ arbitrary units and for lipid peroxidation 0.024 arbitrary units at baseline. Any change of myeloperoxidase activity and of lipid peroxidation was not observed during the study period; analogous results were also noted for the

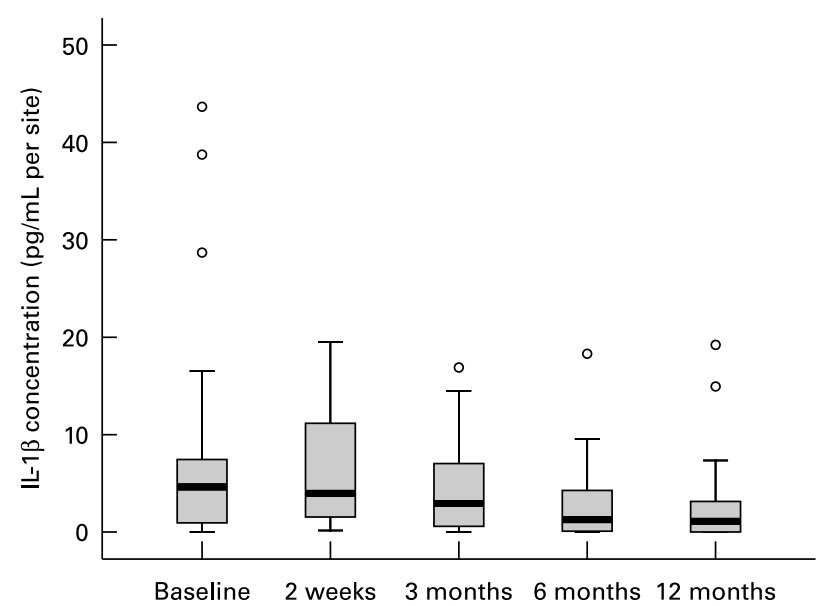

Fig. 1. Concentration of IL-1 $\beta$ in gingival crevicular fluid during the nutritional intervention programme. The central line is the mean; the box represents the lower and upper quartiles; the whisker shows the maximum and minimum values; $O$, outliers. The concentration of IL-1 $\beta$ was significantly reduced after the nutritional intervention $(P<0.001$; Friedman's test). Mean value at 12 months was significantly different from that at baseline $(P=0.004$; Wilcoxon's test). recorded antioxidative variable glutathione peroxidase in stimulated whole saliva (data not shown). After 12 months of the nutritional intervention the activity of catalase showed a tendency to lower values (Fig. 3).

The results of the microbiological analysis of gingival crevicular fluid by real-time PCR are given in Table 4. In $50 \%$ of the patients, Porphyromonas gingivalis was detectable at baseline and only three subjects had a load of more than $10^{5}$ per site. A. actinomycetemcomitans was present in three of twenty subjects. The differences between the dates of examination of the percentages of bacterial counts from the recorded six periodontal pathogens were not statistically significant during the nutritional intervention period.

\section{Discussion}

This is the first study to have focused on the effects of a nutritional intervention programme on clinical, immunological and microbiological variables in patients with chronic periodontitis and the metabolic syndrome, respecting the concept of wholesome nutrition. With professional support of nutritionists, the participants of the study changed their dietary patterns to a low-fat diet, so-called wholesome nutrition. Wholesome

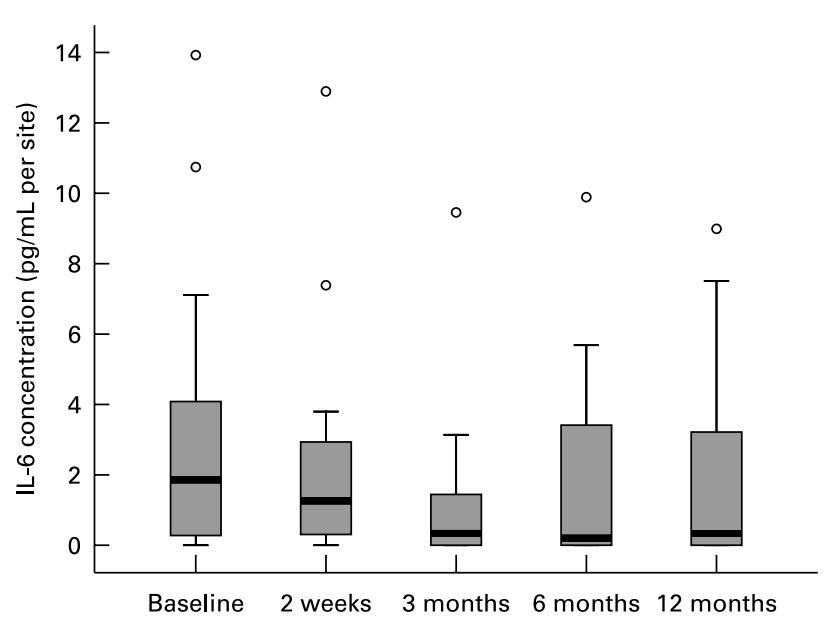

Fig. 2. Concentration of IL- 6 in gingival crevicular fluid during the nutritional intervention programme. The central line is the mean; the box represents the lower and upper quartiles; the whisker shows the maximum and minimum values; $O$, outliers. The concentration of IL- 6 was significantly reduced after the nutritional intervention $(P=0.022$; Friedman's test). 


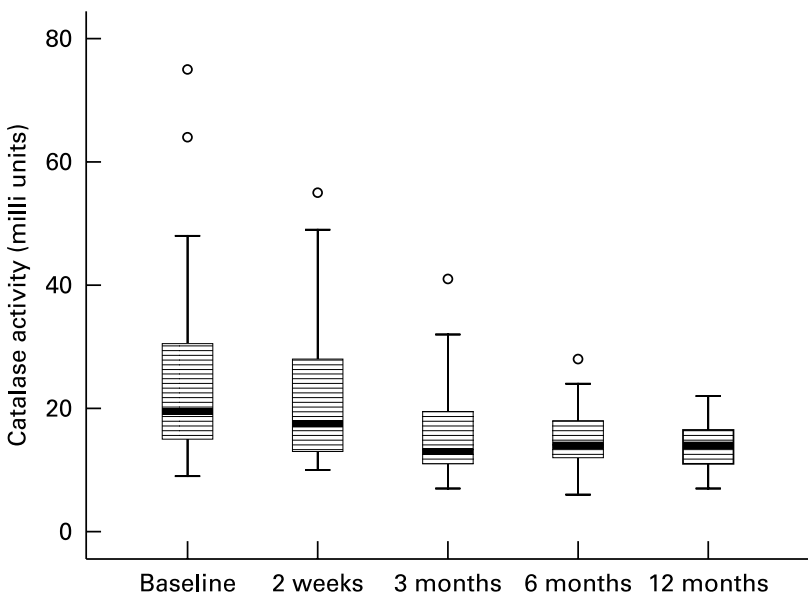

Fig. 3. Activity of catalase during the nutritional intervention programme. The central line is the mean; the box represents the lower and upper quartiles; the whisker shows the maximum and minimum values; $O$, outliers. The reduction during the intervention period is not statistically significant $(P=0.937)$.

nutrition is gaining increasing acceptance as a healthpromoting, sustainable and equitable diet ${ }^{(32)}$. This form of diet is described by Koerber et al. ${ }^{(21)}$ and is mainly composed of vegetables, fruits, whole-grain products, potatoes, legumes and dairy products as well as a limited consumption of meat, fish and eggs. The regular participation at the consultations with the nutritionists demonstrates the participants' high compliance.

Vegetarian-based diets offer a number of nutritional benefits, including lower levels of saturated fat, cholesterol and animal protein, as well as higher levels of carbohydrates, dietary fibre, $\mathrm{Mg}, \mathrm{K}$, folate and antioxidants such as vitamins $\mathrm{C}$ and $\mathrm{E}$ and phytochemicals. Substantial evidence indicates that diets using non-hydrogenated unsaturated fats as the predominant form of dietary fat, whole grains as the main form of carbohydrates, an abundance of fruits and vegetables and adequate $n-3$ fatty acids can offer significant protection against $\mathrm{CVD}^{(33)}$. The effects of diet on the pathogenesis of CVD can be mediated through multiple biological pathways other than serum lipids, including oxidative stress, subclinical inflammation, endothelial dysfunction, insulin sensitivity, blood pressure and thrombotic tendency ${ }^{(34)}$. It is plausible, therefore, that especially through its impact on lipid profile, oxidative stress and inflammation, diet may develop effects on the onset and progression of periodontal diseases.

At 12 months after the nutritional intervention period, the patients showed a significant reduction of probing pocket depth, gingival inflammation and, in accordance with these clinical variables, decreased concentrations of the cytokines IL-1 $\beta$ in gingival crevicular fluid. IL- $1 \beta$ and IL-6 are important proinflammatory cytokines, which mediate the tissue destruction in periodontal disease. The levels of IL-1 $\beta$ and IL-6 are closely related to severity of the periodontitis ${ }^{(35)}$. Expression and release of these cytokines might be also influenced by nutrients ${ }^{(12)}$. Among the nutrients, fats have a large potential for modulating cytokine metabolism. For example, fats rich in $n$-6 PUFA enhance IL- 1 production and tissue responsiveness to cytokines, fats rich in $n$-3 PUFA have the opposite effect, MUFA decrease tissue responsiveness to cytokines and IL- 6 production is enhanced by unsaturated fatty acid intake ${ }^{(36)}$. In contrast to Western dietary patterns, the 
wholesome diet contains a higher intake of PUFA and non-hydrogenated unsaturated fats. $n-3$ Fatty acids are found in high concentrations in certain plant products, for example, linseed and walnut ${ }^{(32)}$. Fish oils, also rich in $n-3$ fatty acids, have been found to inhibit the production of IL-1 $\beta$, IL-6 and $\mathrm{TNF}-\alpha^{(37)}$ and decrease alveolar bone resorption in animal experiments ${ }^{(38)}$. On the basis of these facts the present study suggests that the modulation of cytokine expression may be a biological mechanism responsible for the observed changes in the clinical variables of periodontal disease since other factors with influence on periodontal disease had not been modified.

The inflammatory response, leading to destruction of periodontal tissues, is initiated by micro-organisms, organised in the biofilm. The role of nutrition in the development and composition of the supragingival plaque biofilm is well established. Investigations that suggest an influence on the periodontopathogenic subgingival microflora are still lacking. Nutrient inadequacies may influence oral microbiological ecology ${ }^{(11)}$. In the present study we observed no significant changes in quantity of the periodontal pathogens Tannerella forsythia, Treponema denticola, Porphyromonas gingivalis, A. actinomycetemcomitans, Prevotella intermedia and $F$. nucleatum in gingival crevicular fluid during the nutritional intervention, suggesting that the nutritional intervention has no significant effect on the quantity of the determined periodontopathogens in the subgingival area. It should be mentioned that patients in this nutrition intervention programme had a mild to moderate form of periodontitis, so we found only about $50 \%$ positive for Porphyromonas gingivalis and about $20 \%$ positive for A. actinomycetemcomitans.

As a result of the microbiological challenge, polymorphonuclear granulocytes are activated and release proteolytic enzymes, including elastase ${ }^{(15)}$. Elastase activity correlated with attachment loss and probing depth ${ }^{(39,40)}$. In the present study including only patients with mild to moderate periodontitis, we detected low values of elastase activity and no changes occurred over the intervention period. The mean probing depth from the collection sites in the present study was $3.2 \mathrm{~mm}$ at baseline and $3.0 \mathrm{~mm}$ after 12 months; this may explain the low values.

Another possible link between nutrition, inflammation and periodontitis is the influence of nutrients on the homeostatic balance between reactive oxygen species and the antioxidant defence system. Damage of tissues in inflammatory periodontal disease can be mediated by reactive oxygen species resulting from the physiological activity of polymorphonuclear granulocytes during the phagocytosis of periodontopathogens. Increased gingival crevicular fluid flow in inflammation is related to higher polymorphonuclear granulocyte levels, and gingival crevicular fluid is constantly mixed with saliva. Polymorphonuclear granulocytes release myeloperoxidase from the azurophil granules into oral fluids; this enzyme catalyses the oxidation of chloride and reduction of $\mathrm{H}_{2} \mathrm{O}_{2}$ to form hypochlorous acid ${ }^{(41)}$. This reaction is specifically considered to be relevant in inflammatory conditions.

Increased levels of lipid peroxidation products, caused by reactive oxygen species, may play a role in the inflammation and tissue destruction in periodontitis. In comparison with periodontal healthy subjects, higher levels of lipid peroxidation products were found in whole saliva from patients with periodontitis. After periodontal therapy, the levels of lipid peroxidation products were reduced ${ }^{(42)}$.

Catalase and glutathione peroxidase facilitate the processing of oxidant molecules to harmless by-products. Glutathione peroxidase and catalase are antioxidant enzymes, which are important for the detoxification of $\mathrm{H}_{2} \mathrm{O}_{2}^{(43)}$, but little information relevant to periodontal disease is available. Wei et al. ${ }^{(18)}$ observed no significant differences in whole saliva glutathione peroxidase activities between periodontal-diseased and healthy subjects.

An adequate intake of antioxidants and PUFA may be important for preventing oxidative stress ${ }^{(19)}$. In the present study we found very low values of oxidative and antioxidative enzyme activities and lipid peroxidation products in stimulated whole saliva and between the several dates of examination no changes could be found. So changes in dietary patterns might be developing no effects on the determined variables in whole saliva.

During the nutritional intervention period the aetiological conditions of periodontitis remained constant. The oral hygiene status did not change. The observed slight reduction of the extent of gingival inflammation may be explained through a modulation of the disposition of the host, because alterations by the microbiological exposition could be as far as possible excluded in the present study. So the most probable mechanism responsible for the slight reduction of inflammation with the attendant slight decrease of probing depth and gingival index over the nutritional intervention period could be a reduced production of IL- $1 \beta$ and IL- 6 in consequence of the diet-related modulation of cytokine expression. The present study gives evidence about the causal association between nutritional factors and periodontal diseases. There are some limitations in the present study. Only patients with mild or moderate periodontitis were included and no controls could be considered. Furthermore it should be mentioned that only women with a mean age of 55.0 (SD 10.9) years participated in the present study. Therefore population-level conclusions should be made with caution. It may be concluded from this prospective clinical study that wholesome nutrition in patients with the metabolic syndrome might reduce inflammatory variables in chronic periodontitis and may improve periodontal health. Further studies in a larger population are needed to find out the mechanisms underlying the associations between several properties of the metabolic syndrome, nutrition and periodontitis.

\section{Acknowledgements}

No external funding, apart from the support of the authors' institutions, was available for the present study. The authors declare that they have no conflict of interest.

We thank all those who volunteered for the study. The authors thank Claudia Ranke for her excellent technical assistance. R. P. performed the clinical examinations and collected the samples. A. J. carried out the laboratory work, analysed the data and wrote the manuscript. S. E. supported the laboratory work, collaborated on the interpretation of the results and performed the statistical analyses. H. J. designed the study, participated in the interpretation of the results and provided statistical support. All authors participated in critically revising the manuscript. 


\section{References}

1. Van Dyke TE \& Sheilesh D (2005) Risk factors for periodontitis. $J$ Int Acad Periodontol 7, 3-7.

2. Ritchie SR \& Kinane DF (2003) Nutrition, inflammation and periodontal disease. Nutrition 19, 475-476.

3. Browning LM \& Jebb SA (2006) Nutritional influences on inflammation and type 2 diabetes risk. Diabetes Technol Ther 8, $45-54$.

4. Blignaut JB \& Grobler SR (1992) High fruit consumption and the periodontal status of farm workers. Clin Prev Dent 14, 25-28.

5. Merchant AT, Pitiphat W, Franz M \& Joshipura HJ (2006) Whole grain fiber intakes and periodontal risk in men. Am $J$ Clin Nutr 83, 1395-1400.

6. Sakki TK, Knuutilla MI, Vimpari SS \& Hartikainen MS (1995) Association of lifestyle with periodontal health. Community Dent Oral Epidemiol 23, 155-158.

7. Al-Zahrani MS, Borawski EA \& Bissada NF (2004) Poor overall diet quality as a possible contributor to calculus formation. Oral Health Prev Dent 24, 345-349.

8. Al-Zahrani MS, Bissada NF \& Borawski EA (2003) Poor overall diet quality as a potential risk factor for periodontitis. $J$ Periodontol 74, 1402.

9. Saito T, Shimazaki Y, Koga T, Tsuzuki M \& Ohshima A (2001) Relationship between upper body obesity and periodontitis. J Dent Res 80, 1631-1636.

10. Boyd LD \& Madden TE (2003) Nutrition, infection and periodontal disease. Dent Clin North Am 47, 337-354.

11. Enwonwu CO (1995) Interface of malnutrition and periodontal disease. Am J Clin Nutr 61, 430S-436S.

12. Grimble RF (1998) Nutritional modulation of cytokine biology. Nutrition 14, 634-640.

13. Palmblad J, Hallberg D \& Rossner S (1977) Obesity, plasma lipids and polymorphonuclear (PMN) granulocyte functions. Scand J Haematol 19, 293-303.

14. Miller DR, Lamster IB \& Chasens AI (1984) Role of the polymorphonuclear leukocyte in periodontal health and disease. $J$ Clin Periodontol 11, 1-15.

15. Drugarin D, Onisei D, Koreck A, Negru S \& Drugarin M (1998) Proinflammatory cytokines production and PMN-elastase release from activated PMN cells in the periodontal disease. Roum Arch Microbiol Immunol 57, 295-307.

16. Armitage GC, Jeffcoat MK, Chadwick DE, Taggart EJ, Numabe Y, Landis JR, Weaver SL \& Sharp TJ (1994) Longitudinal evaluation of elastase as a marker for the progression of periodontitis. J Periodontol 65, 120-128.

17. Aruoma OI (1994) Nutrition and health aspects of free radicals and antioxidants. Food Chem Toxicol 32, 671-683.

18. Wei PF, Ho KY, Ho YP, Wu YM, Yang YH \& Tsai CC (2004) The investigation of glutathione peroxidase, lactoferrin, myeloperoxidase and interleukin-1 $\beta$ in gingival crevicular fluid: implications for oxidative stress in human periodontal diseases. J Periodontal Res 39, 287-293.

19. Jenkinson A, Franklin AF, Whale K \& Duthie GG (1999) Dietary intake of polyunsaturated fatty acids and indices of oxidative stress in human volunteers. Eur J Clin Nutr 53, 523-528.

20. Sculley DV \& Langley-Evans SC (2002) Salivary antioxidants and periodontal disease status. Proc Nutr Soc 61, 137-143.

21. Koerber KV, Maennle T \& Leitzmann C (2004) Vollwert Ernaehrung. Konzeption einer Zeitgemaessen Ernaehrungsweise (Full Value - Nutrition. Design of a Modern and Sustainable Food), 10th ed. Heidelberg: Haug.

22. Löe H \& Silness J (1963) Periodontal disease in pregnancy. I. Prevalence and severity. Acta Odontol Scand 21, 533.

23. Turesky S, Gilmore ND \& Glickman I (1970) Reduced plaque formation by the chloromethyl analogue of victamine C. J Periodontol 41, 41-43.
24. Ashimoto A, Chen C, Bakker I \& Slots J (1996) Polymerase chain reaction detection of 8 putative periodontal pathogens in subgingival plaque of gingivitis and advanced periodontitis lesions. Oral Microbiol Immunol 11, 266-273.

25. Rudney JD, Chen R \& Pan Y (2003) Endpoint quantitative PCR assays for Bacteroides forsythus, Porphyromonas ginigvalis and Actinobacillus actinomycetemcomitans. J Periodontal Res 38, 465-470.

26. Fouad AF, Barry J, Caimano M, Clawson M, Zhu Q, Carver R, Hazlett K \& Radolf JD (2002) PCR-based identification of bacteria associated with endodontic infections. J Clin Microbiol 40, $3223-3231$.

27. de Mendez I, Young KR Jr, Bignon J \& Lambre CR (1991) Biochemical characteristics of alveolar macrophage-specific peroxidase activities in the rat. Arch Biochem Biophys 289, 319-323.

28. Davies B \& Edwards SW (1989) Inhibition of myeloperoxidase by salicylhydroxamic acid. Biochem J 258, 801-806.

29. Esterbauer H \& Cheeseman KH (1990) Determination of aldehydic lipid peroxidation products: malonaldehyde and 4hydroxynonenal. Methods Enzymol 186, 407-421.

30. Lawrence RA \& Burk RF (1976) Glutathione peroxidase activity in selenium-deficient rat liver. Biochem Biophys Res Commun 71, 952-958.

31. Beers RF \& Sizer IW (1952) A spectrophotometric method for measuring the breakdown of hydrogenic peroxide by catalase. J Biol Chem 195, 133-140.

32. Leitzmann C (2005) Wholesome nutrition: a suitable diet for the new nutrition science project. Public Health Nutr 8, $753-759$.

33. Hu FB \& Willet WC (2002) Optimal diets for prevention of coronary heart disease. JAMA 27, 2569-2578.

34. Giugliano D, Ceriello A \& Esposito K (2006) The effects of diet on inflammation. Emphasis on the metabolic syndrome. $J \mathrm{Am}$ Coll Cardiol 48, 677-685.

35. Ishihara Y, Nishihara T, Kuroyanagi T, Shirozu N, Yamagishi E, Ohguchi M, Koide M, Ueda N, Amano K \& Noguchi T (1997) Gingival crevicular interleukin-1 and interleukin-1 receptor antagonist levels in periodontally healthy and diseased sites. J Periodontal Res 32, 524-529.

36. Grimble RF \& Tappia PS (1998) Modulation of pro-inflammatory cytokine biology by unsaturated fatty acids. Z Ernahrungswiss 37, 57-65.

37. Wu D, Han SN, Meydani M \& Meydani SN (2004) Effect of concomitant consumption of fish oil and vitamin $\mathrm{E}$ on production of inflammatory cytokines in healthy elderly humans. Ann N Y Acad Sci 1031, 422-424.

38. Kesavalu L, Vasudevan B, Raghu B, et al. (2006) Omega-3 fatty acid effect on alveolar bone loss in rats. J Dent Res $\mathbf{8 5}$, 648-652.

39. Alpagot T, Silverman S, Lundergan W, Bell C \& Chambers DW (2001) Crevicular fluid elastase levels in relation to periodontitis and metabolic control of diabetes. J Periodontal Res 36, $169-174$

40. Gustafsson A, Asam B, Bergström K \& Söder PÖ (1992) Granulocyte elastase in gingival crevicular fluid. A possible discriminator between gingivitis and periodontitis. J Clin Periodontol 19, 535-540.

41. Miyasaki KT (1991) The neutrophil: mechanisms of controlling periodontal bacteria. J Periodontol 62, 761-774.

42. Tsai CC, Chen HS, Chen SL, Ho YP, Ho KY, Wu YM \& Hung CC (2005) Lipid peroxidation: a possible role in the induction and progression of chronic periodontitis. $J$ Periodontal Res 40, 378-384.

43. Ho YS, Magnenat JL, Gargano M \& Cao J (1998) The nature of antioxidant defense mechanisms: a lesson from transgenic studies. Environ Health Perspect 106, 1219-1228. 